\title{
Challenges of diagnosing and treating Charles Bonnet syndrome
}

Maximilian Franke, MD,* Lisa Rauschenberger, MD,* and Felix Fluri, MD

Neurology: Clinical Practice August 2018 vol. 8 no. 4 359-361 doi:10.1212/CPJ.0000000000000484
Correspondence

Dr. Maximilian Franke

franke_m1@ukw.de

Charles Bonnet syndrome (CBS) is characterized by complex visual hallucinations in visually impaired but otherwise psychologically normal individuals. ${ }^{1}$ CBS is primarily diagnosed in patients with an ophthalmic disease but is also seen in stroke patients with hemianopsia. ${ }^{2}$ Treatment of these vivid hallucinations is challenging and there is no standard treatment. Here, we present a stroke patient with CBS successfully treated with melperone.

\section{PRACTICAL IMPLICATIONS}

Consider Charles Bonnet syndrome in patients with optical hallucinations after occipital stroke.

\section{Case report}

An 82-year-old woman with a history of arterial hypertension and diabetes mellitus presented with an acute right-sided loss of vision. Neurologic examination revealed an isolated right homonymous hemianopia but was otherwise normal. This finding was confirmed by an automated perimetry (figure, A). Brain MRI showed an acute ischemic stroke within the left occipital lobe and an old infarction involving the right occipital lobe (figure, B). The patient was neither aware of this stroke nor did she remember any symptoms associated with it. One day after infarction, the patient noticed episodic and simple geometric shapes in the form of ornaments exclusively in her right visual field followed by complex shapes of faces and human bodies moving in a bizarre manner. Laboratory tests ruled out an infectious, toxic, or metabolic cause of these visual hallucinations. An EEG did not show epileptic activity. A psychiatric examination showed the patient fully oriented and without signs of mental illness. These findings allowed a diagnosis of CBS according to the criteria of Gold and Rabins. ${ }^{3}$ We initiated therapy with aripiprazole ( $5 \mathrm{mg}$ daily) to treat the visual hallucinations. However, the 2-month intake of aripiprazole did not significantly reduce the frequency or the intensity of these hallucinations. Therefore, we administered melperone ( $25 \mathrm{mg}$ daily), resulting in a disappearance of the visual hallucinations 2 weeks after initiation of treatment. Three clinical reassessments of the visual fields did not reveal an improvement of the hemianopsia.

\section{Discussion}

To date, no universally accepted diagnostic criteria for CBS exist. Most of the proposed diagnostic criteria require formed visual hallucinations in the psychologically normal elderly patient. ${ }^{1}$ Regarding the pathomechanism of the CBS, 2 main theories are discussed: the perceptual release hypothesis proposes that a normal visuo-sensory input inhibits irrelevant sensations from the conscious perception of images. By reducing visuo-sensory inputs, the threshold to suppress irrelevant sensations is not achieved and subconscious perceptions are released into consciousness, leading to visual hallucinations. ${ }^{4}$ The deafferentation theory suggests that a loss of visuo-sensory input to the visual cortex results in spontaneous neuronal firing and subsequently in abnormal visual perceptions. ${ }^{5}$ In this context, serotonin $2 \mathrm{~A}$ receptors $\left(5 \mathrm{HT}_{2 \mathrm{~A}} \mathrm{R}\right)$, which are highly expressed in the visual cortex, play a crucial role: $5 \mathrm{HT}_{2 \mathrm{~A}} \mathrm{R}$ are

\footnotetext{
*These authors contributed equally to this work.
}

Department of Neurology, University Hospital Würzburg, Germany.

Funding information and disclosures are provided at the end of the article. Full disclosure form information provided by the authors is available with the full text of this article at Neurology.org/cp. 
A
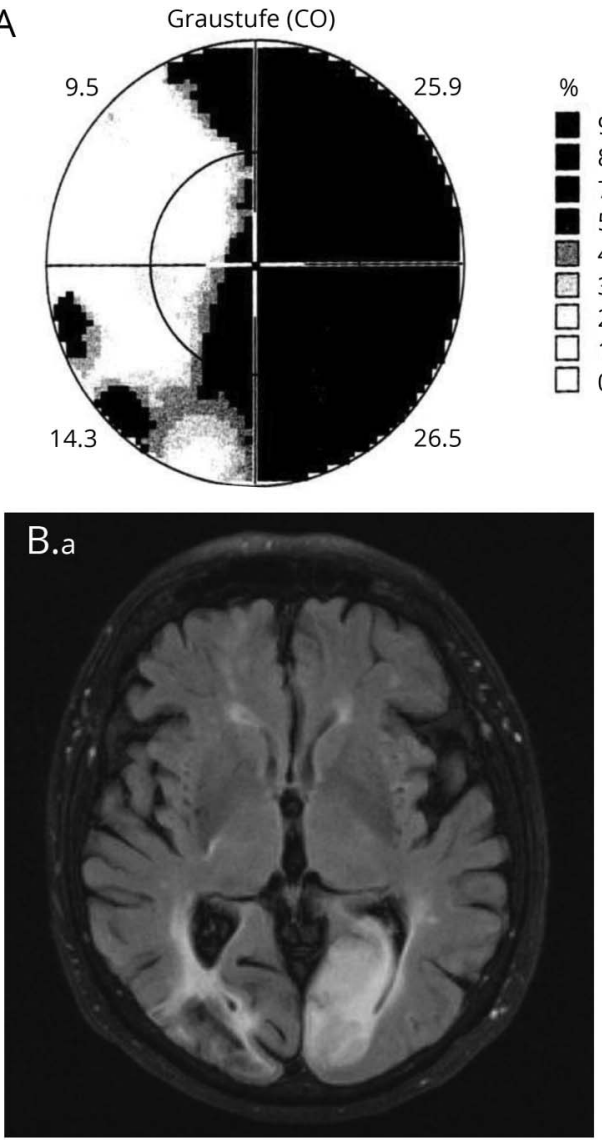
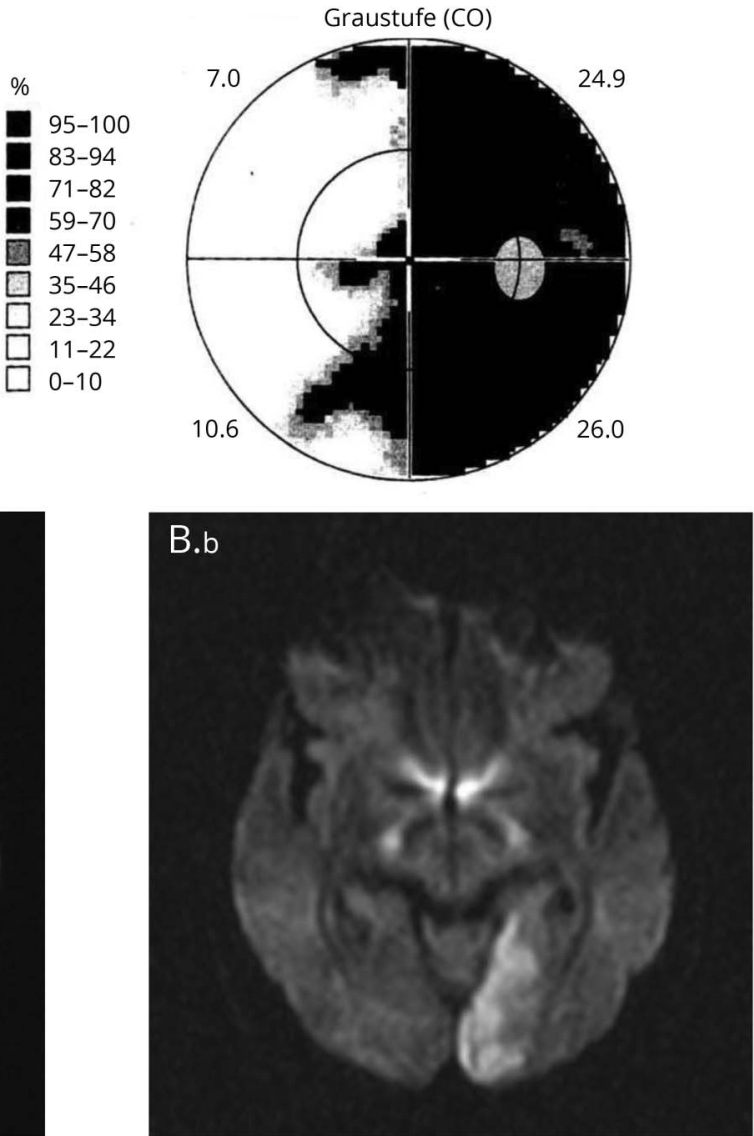

(A) Visual field. The presence of an isolated right homonymous hemianopia was confirmed by automated perimetry for the left and right eye. (B) Brain MRI. (B.a) Representative diffusion-weighted imaging reveals the acute ischemic lesion within the left occipital lobe; (B.b) fluid-attenuated inversion recovery sequence shows both the old ischemic lesion within the right as well as the acute ischemic lesion of the left occipital lobe.

activated in the absence of visual stimuli and increase the excitability of the visual network. This destabilizes neuronal activity, which then results in the formation of visual hallucinations. ${ }^{6}$

The visual hallucinations of patients with CBS are described as either simple geometric patterns or complex recognizable shapes such as faces or floral pictures. The hallucinations are typically seen in the area of the newly acquired vision loss. Even though largely unrecognized in the daily hospital routine, CBS should be considered as a diagnosis of hallucinating patients in whom other causes of visual hallucinations were excluded. Among 120 patients with homonymous hemianopsia and occipital lobe damage, 13\% experienced complex visual hallucination in the affected visual field. ${ }^{7}$ There is no specific therapy for CBS. A mainstay of treatment is the reassurance of patients that these visual hallucinations are inherently benign and not a sign of a psychiatric disorder. A large number of anticonvulsant and antipsychotic drugs (e.g., carbamazepine, valproate, olanzapine, mirtazapine) have been reported to relieve visual hallucinations associated with $\mathrm{CBS} .^{2}$ However, patients with CBS may have spontaneous improvement as well. A study of visually impaired patients who also reported hallucinations that were not treated showed spontaneous remission in $28 \%$ of the patients 1 year after diagnosis, although visual measures did not change. ${ }^{8}$

In the present case, melperone resulted in an improvement of visual hallucinations, which is in line with an observation of a previous case series. ${ }^{9}$ By blocking $5 \mathrm{HT}_{2 \mathrm{~A}} \mathrm{R}$, melperone might attenuate the excitability of the visual network in the absence of visual inputs and thus counteract the deafferentation, which might lead to a cessation of visual hallucinations. This assumption might be further corroborated by the fact that aripiprazole-a partial agonist at the $5 \mathrm{HT}_{2 \mathrm{~A}} \mathrm{R}$ - had no effect on the hallucinations. However, the effectiveness of melperone on hallucinations has to be proved further in a larger cohort of patients with CBS.

\section{Author contributions}

Maximilian Franke and Lisa Rauschenberger: study concept and design, acquisition of data, patient care. Felix Fluri: study concept and design, study supervision. 


\section{Study funding}

No targeted funding reported.

\section{Disclosure}

The authors report no disclosures. Full disclosure form information provided by the authors is available with the full text of this article at Neurology.org/cp.

Received February 23, 2018. Accepted in final form May 2, 2018.

\section{References}

1. Teunisse RJ, Cruysberg JR, Hoefnagels WH, Verbeek AL, Zitman FG. Visual hallucinations in psychologically normal people: Charles Bonnet's syndrome. Lancet 1996; 347:794-797.
2. Ashwin PT, Tsaloumas MD. Complex visual hallucinations (Charles Bonnet syndrome) in the hemianopic visual field following occipital infarction. J Neurol Sci 2007; 263:184-186

3. Gold K, Rabins PV. Isolated visual hallucinations and the Charles Bonnet syndrome: a review of the literature and presentation of six cases. Compr Psychiatry 1989;30 90-98.

4. Cogan DG. Visual hallucinations as release phenomena. Albrecht Von Graefes Arch Klin Exp Ophthalmol 1973;188:139-150.

5. Burke W. The neural basis of Charles Bonnet hallucinations: a hypothesis. J Neurol Neurosurg Psychiatry 2002;73:535-541.

6. Kometer M, Schmidt A, Jancke L, Vollenweider FX. Activation of serotonin 2A receptors underlies the psilocybin-induced effects on oscillations, N170 visual-evoked potentials, and visual hallucinations. J Neurosci 2013;33:10544-10551.

7. Kölmel HW. Complex visual hallucinations in the hemianopic field. J Neurol Neurosurg Psychiatry 1985;48:29-38.

8. Jackson ML, Bassett KL. The natural history of the Charles Bonnet syndrome: do the hallucinations go away? Eye 2010;24:1303-1304.

9. Batra A, Bartels M, Wormstall H. Therapeutic options in Charles Bonnet syndrome. Acta Psychiatr Scand 1997;96:129-133.

\section{Subspecialty Alerts by E-mail!}

Customize your online journal experience by signing up for e-mail alerts related to your subspecialty or area of interest. Access this free service by clicking on the "My Alerts" link on the home page. An extensive list of subspecialties, methods, and study design choices will be available for you to choose from-allowing you priority alerts to cutting-edge research in your field! 